\title{
L'ARTICULATION ENTRE FORMATION ET MONDE DU TRAVAIL ET SA RELATION AVEC LES TRAJECTOIRES ET LES IDENTITÉS DES DOCTEURS SELON UNE APPROCHE SYSTÉMIQUE
}

\author{
Author(s) / Auteur(s) : \\ Miriam APARICIO \\ Docteur en Sciences de l'éducation. Université Paris V, Sorbonne, 2005. \\ Docteur en éducation. Université Catholique Argentine, 1995. \\ Master en éducation, relations humaines et communication. Université Catholique Argentine, 1995. \\ HDR en Éducation, Université Paris X, Nanterre, France, 2007. \\ HDR en Psychologie. Université Lille3, Lille, France, 2007. \\ Conseil national pour la recherche scientifique (CONICET, Argentine / CNRS de la France). \\ Universidad Nacional de Cuyo, Mendoza, Argentina. Professeure des universités depuis 1989. \\ miriamapar@yahoo.com
}

\begin{abstract}
:
This article analyzes the social representations related to the working world that groups of PhDs and PhD students who belong to French and Argentine institutions in the same disciplinary fields have (2005-2009; 20092014). At the methodological level, we have used a specific qualitative technique, hierarchical evocation. This has allowed us to observe how the actors themselves, in their own words, view the process of professional insertion in the workplace, promotion, preservation of human resources, and the growing uncertainty, among other issues, associated with the working world. Likewise, we have been able to capture their projections for the future and their professional expectations, as well as the impact these processes have on their pathways and identities, which many times are fragile and exist on unstable stages, stages where nationalization, liberalism and the socio-political and economic crisis are leaving their mark. To this general framework, we must add other aspects such as the effects of university for the masses on employment, the saturation of degree-holders and, at the same time, the devaluation of degrees, and their background effects. Our results show "contextualized" similarities and differences between the groups. The relationship between individual/context/individual, as well as the "back and forth" between the micro, meso and macro levels, clearly emerge from our comprehensive sui generis systemic perspective, The Three Dimensional Spiral of Sense (Aparicio, 2012, $2015 a, b$ ).
\end{abstract}

\section{Keywords:}

working world, social representations, crisis, PhD/PhD students, systemic sui generis approach

\section{Résumé :}

L'article analyse les représentations sociales concernant le monde du travail des groupes de docteurs/doctorants, appartenant à des institutions françaises et argentines et aux mêmes champs disciplinaires (2005-2009; 2009-2014). Sur le plan méthodologique, nous avons utilisé une technique qualitative spécifique, l'évocation hiérarchisée. Elle nous a permis, à travers des mots des propres acteurs, d'observer comment ils perçoivent le processus d'insertion professionnelle, de promotion, de préservation ou pas de la ressource humaine, de précarisation croissante et d'autres problématiques associées au monde du travail. De même, nous avons saisi leurs projections sur l'avenir et sur les attentes professionnelles ainsi que l'impact de ces processus sur leurs trajectoires et identités, plusieurs fois fragilisées dans des scénarios instables; des scénarios où la mondialisation, le libéralisme et la crise socio-politique et économique, laissent des " traces à feu ». A ce cadre général, il faut ajouter d'autres marqueurs tels que les effets de l'université de masses sur le plan de l'emploi; la saturation des diplômés, et, à la fois, de dévaluation de diplômes, avec leurs effets de plafond. Les résultats nous montrent des convergences et divergences «contextualisés» pour les différents groupes. La relation sujet/contexte/sujet ainsi que les «allers-retours » entre le niveau micro, méso y macro émergent clairement à partir de notre approche systémique-compréhensive sui generis, intitulé Three-Dimensional Spiral of Sense (Aparicio, notamment $2012 ; 2015 a, b$ ).

\section{Mots-clés :}

monde du travail, représentations sociales, crise, docteurs/doctorants, approche systémique sui generis 


\section{INTRODUCTION}

La relation entre formation/emploi et, plus globalement, avec le monde du travail réunit l'interaction $\mathrm{du}$ sujet et de son contexte ; un contexte qui valorise son apport ou non, qui le permet l'épanouissement ou non, en impactant sur les trajectoires et l'identité personnelle, professionnelle et, enfin, institutionnelle et macro-nationale (Dubar, 1991, 1992).

Cependant, encore les théoriciens des carrières «nomades » ou «protéennes » attribuent à l'individu un poids essentiel. Ici nous nous demanderons : Quelle est l'importance relative des contextes; ici du monde du travail ? Est-il possible d'envisager la problématique des trajectoires, des identités et des facteurs associés, interagissant dans un système méso institutionnel et macro social complexe ? Est-il possible d'envisager la problématique des trajectoires, des identités en considérant les représentations partagées des groupes qui ont passé par un doctorat concernant le monde du travail et les caractéristiques qu'il présente actuellement ? Cela suppose une nouvelle épistémologie pour étudier les carrières.

J'essaierai d'apporter des réponses aux questions posées en 2 Parties, en incorporant certains aspects théoriques et d'autres méthodologiques.

Le plan de travail est le suivant :

- partie 1 : j'aborderai deux aspects.

Le premier - esquissé dans les grandes lignes - renvoie à la qualité institutionnelle, une notion multidimensionnelle et qui a donné lieux a des houleux débats ; aussi, aux modèles et critères les plus et les moins traités dans les recherches empiriques (Plus de détail, cf. Aparicio, 2009a).

Le second concerne les axes épistémiques des plusieurs recherches menées dans le cadre du Conseil national pour la recherche scientifique (CONICET, Argentine), effectuées avec diverses populations et techniques depuis 1995 .

- partie 2 : j'aborderai, une nouvelle fois, deux aspects.

Le premier concerne la relation formation / emploi en chiffres. Cela nous permettra d'observer la situation générale des sujets qui ont abouti au $3^{\mathrm{e}}$ cycle.

Le second, renvoie aux recherches comparatives-compréhensives, menées à propos des trajectoires des doctorants/docteurs de institutions françaises et argentines et qui nous ont permis d'approfondir dans le «sens» et les « raisons » qui sont à la base des chiffres des systèmes méso et macro nationaux.

De toutes les recherches réalisées avec des docteurs, nous nous arrêterons ici dans la première (Cnam et Universidad Nacional de Cuyo) ; recherche celle-ci qui a obtenu le Prix de la Marie de Paris (cf. Rapport Marie de Paris, Aparicio 2009b).

\section{CADRE THÉORICO-MÉTHODOLOGIQUE ET LES RECHERCHES MENÉES}

\section{PARTIE 1}

\subsection{L'évaluation de la Qualité}

Pour comprendre le pourquoi de cette option épistémologique systémique, nous nous arrêterons un moment sur la situation trouvée par l'auteure en matière de recherches concernant à éducation ou à travail en 1995 ; recherches qui n'aboutissaient pas encore à une approche intégratrice.

Plus précisément, à ce moment, l'Évaluation de la qualité (formation et emploi) est initiée en Argentine. L'amélioration de la qualité à l'université et son articulation avec le contexte commence à constituer une priorité. Les politiques universitaires mettent l'accent sur la nécessité d'aboutir à des analyses systémiques. Cependant, l'évaluation reste réduite aux chiffres, aux inputs et ouputs mais les processus humains, qui sont à la base de ces chiffres, demeurent inconnus dans notre système national 
et, plus globalement, dans le cadre international (Aparicio, 2010, 2012). En effet, les études, descriptives (\%), nous montraient des diagnostics de situation mais elles ne faisaient pas référence à des causes /conditions / processus / «sens » sous-jacents aux statistiques (universités et entreprises). Les modèles employés, bien qu'appelés systémiques, suivant une stratégie pas innocente, évaluaient seulement des résultats secondaires et macro, laissant de côté la compréhension pour privilégier la description et les diagnostics vides de sens.

\subsection{Axes épistémiques}

Mettre en place une analyse systémique des facteurs qui impactent sur la qualité institutionnelle et sur la réussite au niveau macro ou méso-organisationnel et, à la fois, de l'impact des contextes macro ou méso-organisationnels sur les sujets, impliquait donc un défi.

Nos recherches vont s'orienter dans cette direction, c'est-à-dire, à la recherche d'une approche plus holistique et intégrative pour analyser les «mécanismes » et/ou facteurs qui anticipent le succès au niveau psycho-individuel, organisationnel et, à moyen terme, macro. Autrement dit, la compréhension des résultats macro et méso-organisationnels (système universitaire et de l'emploi) a été faite en abordant les processus micro psychologiques et méso-institutionnels.

Cette nouvelle approche - qui cristallisera dans la production scientifique $(2005,2007$ jusqu'à 2015 a, b) - réunit explication et compréhension, méthodologie quantitative et qualitative, stratégie d'analyse macro-micro-méso-macro, processus et résultats.

Nos modèles, de circuit ouvert, supposent un double sens ou «aller-retour » entre l'individu et son contexte institutionnel avec des effets tant positifs comme négatifs sur les sujets et sur les institutions. Dans les divers recherches complémentaires effectuées, qui couvrent quatre décennies, nous avons intégré, aussi, la voie diachronique et synchronique ; la voie rétrospective avec la voie prospective : l'histoire des sujets et des organisations avec leurs cultures particulières anticipant les trajectoires ; la voie théorique et la voie des pratiques visant au transfert.

Ces trajectoires et les problématiques associées, reviendront sur le système méso et sur individu luimême : voire, un individu plus comblé ou plus frustré à cause du manque de reconnaissance d'une organisation ; possédant une identité plus forte ou plus faible au sein d'un milieu qui ne lui serait pas toujours favorable ; un individu possédant de niveaux plus hauts ou plus bas de coping et de résilience, ce qui permettra de faire face aux obstacles qui présente le monde du travail; ayant des représentations plus ou moins positives sur l'importance actuelle de l'éducation en tant que canal du progrès sous un scénario de crise socio-économique; ou possédant des attentes plus ou moins favorables par rapport à l'avenir.

$\mathrm{Au}$ milieu de la problématique des trajectoires émerge, tout au fond, le besoin de formation des compétences et, surtout, des compétences «sociales», un aspect essentiel aujourd'hui, selon nos recherches, au moment de l'explication et de la compréhension des divers parcours universitaires et professionnels des docteurs/doctorants et du niveau du succès ou d'échec connu.

\section{PARTIE 2}

Arrêtons-nous un moment sur quelques chiffres qui montrent la relation entre formation disciplinaire et emploi.

\subsection{La relation formation/emploi}

Les références seront brèves, tout simplement pour montrer l'influence qui exerce un niveau plus haut d'instruction sur le plan de l'emploi et le décalage pouvant exister entre ces deux entités ${ }^{1}$.

La valeur du diplôme: globalement considéré, l'accès à l'enseignement supérieur devient plus courant. En France, depuis 1980, le nombre de sortants de l'enseignement supérieur court a doublé, celui du supérieur a quadruplé. Ainsi, déjà en 1998 déjà environ d'un sortant du système éducatif sur

\footnotetext{
${ }^{1}$ On renvoie aux ouvrages du Céreq et au Rapport présenté à la Ville de Paris.
} 
deux est issu de l'enseignement supérieur (Aparicio \& Cros, 2015c; cf. Cereq). Cependant, l'obtention d'un diplôme - même si elle reste la clé essentielle au milieu de la problématique de la dévaluation des diplômes - présente des différences et tendances plus spécifiques par filières et institutions. La valeur du diplôme est associée donc à la qualité institutionnelle (histoire, image dans la société, rankings,...). Le même phénomène a été observé en Argentine : les diplômés se placent mieux sur l'échelle hiérarchique et ils valorisent bien son diplôme. Mais ceux qui s'insèrent dans les échelons les plus hauts de la hiérarchie professionnelle proviennent, en général, des institutions qui certifient la qualité, assurant un meilleur avenir professionnel (Aparicio, 2004a, 2004b).

De plus, en France, les diplômés d'écoles de commerce et des écoles d'ingénieurs sont le moins affectés par le chômage, occupant plus souvent des emplois de cadres et des statuts plus stables. Plus globalement, les sortants de l'enseignement supérieur ont été rarement au chômage, presque la moitié des jeunes trouve leur premier emploi immédiatement et les emplois non temporaires dominent (notamment, sortants d'écoles d'ingénieurs ou de commerce).

Les salaires et le genre: les salaires sont associés aussi à la hiérarchie des diplômes et prestige institutionnel, s'observant encore des différences salariales entre hommes et femmes, quel que soit le diplôme de sortie (Aparicio \& Cros, 2015c).

L'entrée dans la vie professionnelle et le chômage : les sortants d'écoles d'ingénieurs et des écoles de commerce présentent une entrée dans la vie active particulièrement favorable. Le taux de chômage pour ces sortants est parmi les plus faibles, ils occupent des emplois non temporaires et la plupart des sortants atteint le statut de cadre.

Par rapport aux docteurs - populations ici abordée -, on observe des différences importantes selon les spécialités et bien qu'ils constituent la population des plus diplômés, l'entrée dans la vie active des sortants de doctorat diplômés ou nom, n'est pas plus facile que pour l'ensemble des diplômés de l'enseignement supérieur. Cependant il existe une variabilité entre spécialités : le taux de chômage est pratiquement inexistant dans le domaine de la santé et plus haute pour les sciences humaines et sociales (shs), notamment, en art, en psychologie et en histoire-géographie. Quant aux salaires, ils sont plus bas que pour les ingénieurs et sortants des écoles de commerce. Enfin, les sortants sans le diplôme sont sanctionnés sur le marché du travail.

La situation, seulement esquissée, et les chiffres des systèmes nationaux - sur lesquels nous ne nous arrêtons pas car cela dépasse notre objectif ici - mettent en évidence la relation entre le niveau de formation et le positionnement dans le monde du travail, même si la question de la dévaluation des diplômes continue au centre de l'agenda politique.

Cependant, dès notre perspective et plus delà de l'intérêt qui offrent les tendances, les rankings, l'analyse de données secondaires ne nous permet pas de connaître quels sont les facteurs qui sont à la base de la situation effective dans le marché. En ce qui nous concerne, ne nous permet pas d'expliquer les causes des trajectoires ni, non plus, de comprendre les raisons profondes des différences observées, placées au niveau des acteurs.

Sur le plan épistémique, le cadre montre bien que, même s'il existe des aspects communs qu'on peut constater, il y a, aussi, d'autres phénomènes qui ne peuvent pas s'expliquer à la lumière des résultats trouvés sur le marché. Par exemple, on assiste à ce moment à une basse mondiale de l'immatriculation dans les filières de génie. Comment expliquer cette situation étant les sortants de génie les mieux placés dans le monde du travail ? A ce moment, nous travaillons dans deux projets pour essayer de « comprendre » cette situation en cherchant les « raisons » des comportements des sujets qui s'insèrent ou pas dans ces filières mais nous n'avons pas encore de réponses. Un cadre est clair : il n’y a pas de comportements ni, non plus, d'effets linéaires où intervient l'homme, avec sa liberté, ses préférences, ses décisions personnelles. Un homme, de plus, placé dans un contexte que ne le détermine pas mais qui impose des contraintes structurelles en conditionnant les choix professionnels. Les «allersretours » des systèmes imbriqués dans ce qui comporte la relation formation-emploi, produisent des effets pervers, pas cherchés ni souhaités mais qui influent sur les choix, sur les trajectoires associés et, même, sur les identités professionnelles, plus frustrées ou plus réalisées (Aparicio, 2007, 2012).

Bref : les statistiques toutes seules ne sont pas suffisantes. Il faut approfondir dans le « sens ». 


\subsection{Les docteurs/doctorants et leurs représentations sur le monde du travail}

\subsubsection{Aspects théoriques}

\subsubsection{Antécédents}

Les recherches avec des docteurs ont été précédées par plusieurs d'autres - centrales et périphériques - , menées avec différentes populations, en utilisant différentes techniques ${ }^{2}$ et en construisant des modèles qu'incorporent cinq facteurs: de base, socioculturels, pedagogico-institutionnels, organisationnels structurels et psychosociaux. Nous arrêter dépasser notre objectif ici. Seulement est important de tenir en compte qu'elles couvrent entre 30 et 40 années de la vie des institutions de notre milieu.

Cette couverture temporelle et spatiale large de nos recherches est important dans le cadre d'une approche systémique. En effet, elle nous a permis : a) d'analyser la relation méso/micro (organisations et individus) au moment d'observer les trajectoires et sa relation avec les identités ; b) de saisir les différences entre les trajectoires des diplômés plus anciens, qui accèdent à l'Université à un autre moment de notre histoire et qui voient mieux cristalliser leurs ambitions que les diplômés récents et actuels. Le «va-et-vient » du système sujet-structure apparait évident (plan macro/micro). c) D'autre part, le fait de travailler dans les mêmes contextes organisationnels mais avec des données issues de différents moments historiques, économico-sociaux et éducatifs, nous a permis de mieux connaître le poids du plan macro sur le plan meso-organisationnel en ce qui concerne l'insertion et la promotion. Les conjonctures ne vont pas de soi, elles laissent sa trace sur les parcours.

\subsubsection{Les effets de la crise dans le monde du travail sur les représentations des plus diplômés : le jeu du sujet et du contexte}

En ce qui concerne la population des docteurs/doctorants français et argentins - objet de cet article nous essayons de connaitre les effets visibles et invisibles des crises, globalement considérées, au niveau de la formation et les nouvelles exigences mais, aussi, au niveau socio-économique, socioculturel et structural jusqu'à toucher le niveau micro-psychologique.

Nous travaillerons, seulement, sur le plan qualitatif et en abordant un noyau : Le monde du travail, même si la recherche a été, aussi, quantitative (cf. analyse descriptive et corrélationnelle de chaque variable par filière et des variables entre soi, Aparicio, 2009-2010. Rapport Prix Ville de Paris).

\subsubsection{Les objectifs de la recherche}

\section{Généraux}

a) Connaître sur le plan qualitatif et quantitatif les facteurs qui influent sur la réussite professionnelle des docteurs / doctorants (français et argentins); b) connaître et comparer (plan descriptif) leur problématique sociologique, psychosociale et institutionnelle en considérant que l'insertion professionnelle est aujourd'hui un problème international (conditions du malaise généralisé dans les institutions de formation au moment de l'entrée dans le métier et après, à l'intérieur des organisations).

Spécifiques du noyau analysé : les représentations sur le monde du travail

a) Connaître les représentations des deux groupes (français et argentin) sur la valeur du travail, du diplôme universitaire, d'une formation doctorale, des chances dans l'avenir, des conditions concrètes d'exercice quotidienne du métier et d'autres aspects, qui pourraient conditionner les possibilités de réussir ; b) donner aux décideurs du domaine éducatif et productif des éléments de réflexion pour la mise en place de programmes d'amélioration de la qualité de la formation, de la formation continue et des conditions de travail dans des organisations.

\subsubsection{Les questions-axes}

Spécifiquement, dans ce qui nous concerne dans cet article, nous essayons d'observer: Quelles représentations ont les docteurs/doctorants concernant l'éducation / la formation doctorale comme

\footnotetext{
${ }^{2}$ Parmi elles : des enquêtes, entretiens semi-directifs, tests, échelles d'évocation hiérarchisée, différentiels sémantiques, paradigme des juges, paradigme d'identification, analyse léxicométrique, évocations hiérarchisées.
} 
vecteur de progrès ? Comment voient-ils le monde du travail aujourd'hui ? Comment voient-ils leur avenir ? Quels sont pour eux les facteurs priorisés par le marché au moment de l'insertion : les savoirs disciplinaires ? L'expérience ? Ces groupes (français et argentins) sont-ils une communauté réelle de membres partageant des «identités » et des «intérêts» spécifiques? Existe-t-il de différences nationales?

Tous ces éléments sont centraux dans la configuration de l'identité professionnelle (Silva \&Aparicio, 2015). Nous avons trouvé, comme nous l'analyserons, des différents scénarios selon filière, discipline, institution, pays. Chacun présente de problématiques différenciées et a besoin de réponses « pertinentes ».

\section{LA METHODOLOGIE}

Elle a été notamment qualitative mais aussi quantitative.

\subsection{Population}

Elle a été constitué par des sujets qui ont passé par une formation doctorale (2005-2009 ; 2009-2014) en France (Cnam, 3 filières : Sciences de la gestion, Formation des adultes et Sociologie du travail) et Argentine (Universidad Nacional de Cuyo, 2 filières : Éducation et Sciences sociales/Sociologie). Dans un second temps, nous avons incorporé à la recherche à une université française de province pour analyser, précisément, la relation sujets/contextes.

\subsection{Techniques}

Elles ont été quantitatives et qualitatives : a) enquête semi-directive avec des phrases ouvertes pour laisser parler les acteurs ; b) entretiens, c) évocations hiérarchisées. La dernière a été la technique fondamentale pour approfondir sur le plan de représentations centrales (nœud/cœur de la représentation) et périphériques (Abric, 1991 ; adaptation de Aparicio).

Dans chaque noyau et à partir des mots dits par nos sujets, nous avons distingué des dimensions/catégories. Nous avons travaillé en 2 temps : dans le premier, nous avons tenu en compte, seulement, la saillance; dans un second temps, nous avons combiné saillance et importance. En appliquant les formules respectives, nous avons construit 4 quadrants (P1, P2, P3 et P4) qui réunissent les représentations de haute fréquence/haute importance $(\mathrm{P} 2=$ le cœur ou nœud de la représentation ; c'est-à-dire, où sont placées les représentations les plus saillantes et les plus importantes); le quadrant de basse fréquence/basse importance (P3). Ici sont placées les RS très peu significatives pour notre analyse. P4 et P1 sont, finalement, les quadrants qui vont réunir les RS de basse fréquence/haute importance et a l'inverse.

Figure 1 : Le nœud et dimensions périphériques des représentations sociales

\begin{tabular}{|l|l|}
\hline P1 & P2 \\
\hline P4 & P3 \\
\hline
\end{tabular}

\section{LES RÉSULTATS}

Nous présentons les résultats selon dimensions construites :

- Dimension organisationnelle, socio-politique et structurelle

- Dimension éducative contenant la formation, le travail et les effets sur le sujet

- Dimension économique recouvrant les attentes face au travail et à l'avenir

- Dimension interpersonnelle et compétences associées

- Dimension personnelle socioaffective et compétences associées

Analysons les aspects centraux. 


\section{La dimension « organisationnelle, sociopolitique es structurelle »}

Dans les trois filières du Cnam, cette dimension est la seule placée au cœur de la représentation concernant plus précisément le monde du travail (P2).

Nous rencontrons deux aspects dont le négatif est fort. Les mots parlent d'eux-mêmes : « difficile», « exploitation », «esclavage », « injustice», « désavantages », « exigences », «production », « technique », « marché », « travail », « usine », « horaires ». C'est un monde « complexe », « principe de réalité ». C'est aussi «conflit», «société», «idéologie», «politique », « crise », « individualisme », « sélection »; ces derniers mots, les plus fréquents dans la filière de Sociologie du travail, révèlent une forte appartenance disciplinaire.

En plus apparaissent des termes comme «jeu politique », «chômage », «crise », «bataille», « pouvoir », « aliénant», « déception», « inégalitaire », « inéquitable », « dur »; un monde qui regarde «l'efficacité » et qui se caractérise par des « horaires rigides » et, plus globalement, par un manque de «flexibilité ».

L'aspect positif est plus marqué chez nos sujets de sciences de gestion, à travers des mots comme «emploi », « projet», « qualité », « activité », « motivation»; c'est un « monde important», « création », « cheminement personnel », « choix », « domaine important de la vie »; « carrière »; ce dernier mot est le seul qui apparaît dans les trois filières françaises et très lié à notre axe d'analyse : l'avenir des docteurs/doctorants dans le monde du travail, leur positionnement, leur mobilité, etc.

$\mathrm{Si}$ nous comparons ces mots avec les mots cités par rapport aux catégories «Université » et «Formation doctorale», nous notons que se manifeste un regard bien différent: la «formation doctorale » est, pour ces docteurs/doctorants, «passionnante », « fondamentale », « riche », « débat », «développe le doute», «provoque l'érudition», «formation de haut niveau», «excellence», "pensée et langage élaborés, soutien », même si la facette négative de la Formation doctorale se révèle sous un autre visage ("dure », «un jeu de pouvoir», un «invention», "pénible », «un problème »). En ce qui concerne le noyau «Université », cet aspect négatif s'est déjà observé comme «place de la haine », des «privilèges », « infantile».

En observant les caractéristiques attribuées par les doctorants/docteurs au Monde du travail et au Monde académique, on note qu'elles sont bien différentes, avec des perceptions, globalement attribuées, au monde du travail dans l'imaginaire collectif (place de «l'exploitation», de «l'injustice», de «l'esclavage »...).

Si nous regardons les réponses des doctorants/docteurs à l'UNCuyo, les mots les plus souvent cités sont «complexité », «difficile»; un «monde qui exige», «égoïste», « injuste»; «amer», « apathique », «fermé », " peu flexible/horaires », « espace où domine une vision ». La place du « conflit», de la «bureaucratie », de «l'incertitude», de la «spéculation», « de l'envie » et du « manque de l'éthique ». Un monde « inégalitaire », « désorganisé », « dissocié »; « un mal de tête »; c'est « une praxis », «syndicalisme », «mondialisation ». C'est l'espace de la "légitimité », de la «dépersonnalisation ». C'est aussi «l'espace de la production» et de la «reproduction sociale», diront les docteurs/doctorants de Sciences sociales. Une nouvelle fois, la facette disciplinaire émerge.

On trouve à côté, pour désigner le monde du travail des mots positifs comme «qualité », « dignité », « espace communautaire», «réalisation de l'existence » et «lieu de l'exercice de la vocation ». C'est un « champ solidaire », « domaine de réalisation », « excellence », « travail ». C'est l'espace du « bien commun », de « l'espoir », de la « diversité »; c'est une « manière de vivre », une « réalité ».

Mais dans les cinq filières, l'image négative domine. Les représentations partagées par tous les interviewés révèlent des préoccupations actuelles importantes par rapport au monde du travail, dans les deux institutions et les deux pays; il n'existe pas de différences marquées entre les filières françaises et argentines.

En ce qui concerne la dimension «éducative» contenant la formation, le travail et les effets sur le sujet, celle qui relie la formation avec le monde du travail, deux aspects sont à noter : d'une part, la vision de cette relation est positive, à certaines exceptions près et, d'autre part, les mots nommés sont proches des mots qui apparaissent par rapport aux représentations de l'Université et de la Formation 
doctorale : les deux mondes trouvent ici un point de rencontre. Les mots les plus souvent nommés sont ici (dans les cinq filières) « compétence » et « développement du potentiel humain ».

Nous pouvons nous en étonner. Les représentations de notre population des cinq filières des deux pays coïncident exactement. La même situation avait été observée à propos des représentations relatives à l'Université et à la Formation doctorale mais dans ce cadre, la « recherche » - noyau dur de la formation doctorale et, à un moindre degré, de l'université - est déjà placée en périphérie des représentations. Il semble que ce soit la même chose à propos de la formation.

Cependant, même si l'image de ces groupes est consistante et proche, elle est faible par rapport aux représentations placées centralement. Elle restera donc située à la périphérie (P3).

La dimension « économique » (attentes/avenir recouvrant les attentes face au travail et à l'avenir) : comme nous l'avons déjà souligné, est la grande absente de la catégorie Formation doctorale, très éloignée des représentations concernant le progrès, le futur. Autrement dit, les doctorants/docteurs n'ont pas beaucoup d'attentes vis-à-vis de leur formation au moment de se lancer dans le monde du travail.

Et pourtant, quand nous analysons ce qui concerne le Monde du travail, des représentations se manifestent. Mais, face à la centralité de la dimension institutionnelle/organisationnelle/structurelle qui concentre la plupart des représentations, ces représentations du Monde du travail perdent de l'importance en se trouvant dans le quadrant 3 du tableau (P3). Il s'agit d'une dimension périphérique.

Les mots clés associés sont ici « salaire », «profits », « concurrence/compétitivité ». L'idée d'un futur meilleur, médiatisé par la formation, n'existe pas. Il faut souligner que le mot « hasard » est cité pour la première fois : quand il s'agit du Monde du travail, où «fonctionnent » bien les normes sociales d'évaluation (partagées et subjectives), le hasard semble jouer un rôle plus important dans l'histoire des sujets.

Si nous comparons avec les représentations des argentins, nous observons d'emblée qu'il existe une convergence forte en ce qui concerne la relation formation-travail. Cependant, la perspective des argentins est toujours un peu plus positive que celle des français quoique encore faible, avec d'autres mots : «opportunité », «promotion», «futur». Cela est consistent avec l'idée dominant dans l'imaginaire argentin; un pays d'une immigration européenne très forte des caractéristiques spéciales dans le siècle passé : il s'agit d'une immigration acceptée et favorisée par les politiques macronationales. Ce fait, unit a la présence d'une université toujours gratuite et un temps de croissance économique unique dans l'histoire argentine, a permis aux immigrants de connaitre la réussite au niveau économique mais, aussi, au niveau de l'éducation. En effet, les immigrants souhaitaient pour leurs enfants qu'ils arrivent à l'université, en regardant l'Université comme le canal de progrès et d'intégration; de promotion, lié à un futur meilleur. Cette représentation collective persiste encore dans le pays; en revanche, en France cette dimension de progrès lié au diplôme de docteur, n'émerge pas à travers des mots dits. Cette faiblesse fera que la dimension dans cette recherche, reste dans le secteur des représentations les moins centrales (P3).

Quant à la dimension « relationnelle » : elle est placée à la périphérie pour les cinq filières (P3). Ce fait est un peu étonnant dans un contexte institutionnel et organisationnel qui priorise les compétences interpersonnelles pour réussir. Mais ce silence peut masquer un aspect caché ou dilué pouvant faire penser à la perte de force de ces relations humaines dans les circonstances actuelles du monde du travail. Même si la dimension ne préoccupe pas les docteurs/doctorants face au monde du travail, deux mots clés apparaissent : identité et reconnaissance, dans le sens d'un «manque de reconnaissance », avec des « identités » fragilisées.

Nous observons bien l'interaction contexte/sujet.

La dimension «socio-affective et les compétences liées »: elle est située à la périphérie des représentations dans les trois filières du Cnam (P3) ; en revanche, elle se situe dans le centre des représentations à l'UNCuyo $(\mathrm{P} 2)$. Dans les cinq filières, trois mots renvoient à trois représentations centrales du monde du travail : «réalisation», «responsabilité », «reconnaissance ». Une seule 
exception : «le sens de l'effort » émerge surtout chez les argentins, associé à «l'ethos culturel » et la présence des immigrants.

Même si le niveau de centralité des représentations change, si nous comparons les données pour les filières du Cnam et celles de l'UNCuyo, la convergence sur le plan des mots les plus cités est forte. Cela montre un cadre similaire des représentations sociales - dans notre cas, partagées par les deux groupes nationaux des cinq filières - par rapport au monde du travail et à ce que les sujets attendent et/ou rencontrent dans leur travail quotidien.

En d'autres termes, même s'il existe quelques différences, surtout on perçoit des aspects plus positifs parmi les argentins dans les dimensions "affective" (plus de reconnaissance) et "économique"; les mots les plus souvent cités renvoient à des convergences.

Cela fait penser à l'existence d'un imaginaire très consistant et répandu par rapport au Monde du travail. Bien que vécus dans deux scénarios socio-politiques et économiques différents, ils sont plus proches au niveau des conceptions et des représentations partagées que ce qu'on aurait pu imaginer.

Le Monde du travail concentre les représentations les plus fréquentes dans la dimension organisationnelle, structurelle et socio-politique, avec des caractéristiques spéciales. Et là semble se manifester fortement la partie négative de ce monde: un monde sauvage, sans pitié, un monde inégalitaire, difficile, désorganisé, fermé, bureaucratique, amer, aliénant, un espace d'injustice, une jungle... Très peu de sujets possèdent une image positive du Monde du travail, comme un espace intéressant, fait d'espoir et de réalisation.

Faisant un bilan :

\subsection{Résultats sur le plan théorique}

La lecture effectuée à partir de mots des propres sujets nous a permis d'observer :

a) Des convergences et divergences traversant les premières expériences des doctorants/docteurs en France et en Argentine (plan psychosocial, sociologique, institutionnel). Parmi celles-ci : différences par filière, par discipline, par unité académique/laboratoire, par institution, par pays. Les facteurs /dimensions qui permettent de comprendre la situation, étant plusieurs fois très similaires. Une question se pose : homogénéisation institutionnelle ? Disciplinaire ? Culturelle ?

b) Même si dans le noyau qui nous occupe, le Monde du travail, les «compétences cognitives » n'émergent pas; il est important de souligner qu'il existe une différence entre les représentations partagées des français et des argentins par rapport à la valorisation des compétences pour agir actuellement : en France, globalement, sont priorisées les compétences sociocognitives ; en Argentine, plutôt, les compétences socio-affectives (typiquement valorisées dans les scenarios latino-américains).

b) Des différences sur les axes qui concernent les identités : en France, le manque de reconnaissance est la première dimension qui «fragilise » l'identité professionnelle; en Argentine, est le manque de crédibilité politique généralisée (cela nous montre une représentation très répandue parmi les argentins, notamment dans les derniers temps et après les évènements politiques diffusés dans le monde).

c) Des différences sur la valorisation de la dévaluation des diplômes : les effets de "plafond» émergent plus clairement en France : plus de docteurs, moins d'attentes sur les possibilités qui apporte un diplôme. En Argentine, au contraire, encore un diplôme de docteur en shs a une valeur un peu plus marquée car la quantité des docteurs dans ce champ disciplinaire diminue (les doctorats en sciences sociales et humaines ont été fermés, globalement, pendant la dictature).

d) Des différences sur le plan de la mobilité, plus marquée en Argentine ; c'est qui émerge dans la dimension économique et la dimension éducative : la formation de $3^{\mathrm{e}}$ cycle encore est liée à un meilleur positionnement socio-professionnel. Autrement dit, la compréhension des problèmes vécus, 
parait influer sur les possibilités de progrès dans le domaine professionnel, sur les trajectoires et identités ${ }^{3}$.

e) Globalement, le scénario que nous venons de décrire montre des caractéristiques répandues par rapport au monde du travail; un monde que pas toujours contribue au développement personnel et professionnel; pas toujours contribue à la construction des trajectoires réussies. Les résultats sont préoccupants : ils nous montrent un visage «peu humain» du monde du travail, qu'impacte - selon les mots des docteurs/doctorants - sur le plan de la réalisation et du progrès ; sur leurs trajectoires.

Dans ce cadre, notre approche dynamique s'avère d'intérêt pour analyser les facteurs qui impactent, précisément, sur les trajectoires et les identités. En fait, l'approche suppose deux pôles : un sujet et un contexte qui jouent interactivement son jeu. Et tant les trajectoires comme les identités se construisent entre ces deux pôles: les sujets (plan biographique) et les contextes (plan relationnel). Chaque trajectoire, comme chaque identité, est donc le fruit du jeu entre le biographique et l'espace de l'interaction; entre un sujet et leur contexte immédiat (organisationnel) et médiat (macronational/social). Dans ce scénario, la rupture de l'identité professionnelle s'accompagne d'une rupture dans la trajectoire et de la mise en œuvre des stratégies identitaires pour survivre dans des contextes difficiles. Trajectoires et identités sont, au final, un point de convergence entre les dimensions individuelle / situationnelle / écologique; entre le soi et les autres, toujours en interaction soutenue.

\subsection{Résultats sur le plan des pratiques}

Les décideurs pourront trouver à partir des résultats, la possibilité d'une meilleure prise de conscience des facteurs liés au monde du travail qui entravent les possibilités de développement personnel et institutionnel.

En effet les cinq groupes présentent des problématiques partagées et, à la fois, différentes ; les cinq nous montrent le besoin de réfléchir sur la nécessité de rénovation/mutation/transformation/innovation institutionnelle/organisationnelle (plan de l'intervention) pour favoriser l'articulation entre formation et emploi ainsi que les conditions de travail. Et cela, visant pas seulement à l'amélioration de deux systèmes en soi mêmes mais, aussi, à la qualité de vie dans le travail et hors travail face à une crise de valeurs, un fatalisme de plus en plus répandu, des niveaux de maladies associées aux conditions de travail où manque, bien souvent, le respect mutuel.

\section{RÉFÉRENCES}

ABRIC, J-C. (1991). Prácticas sociales y representaciones. Coyoacán (DF., Mexique).

APARICIO, M. (2004a). Réussite, exclusion et logiques associées dans le système productif anticipé par le système éducatif. La personne et ses rapports au travail (59-63). In: A. Lancry \& C. Lemoine. L'Harmattan (Paris, France).

APARICIO, M. (2004b). El rol de las organizaciones y su relación con logro y satisfacción en universitarios. Un análisis desde el modelo "consumo-inversión”. Revista de Psicología Social y Personalidad, 20(1): 1-22.

APARICIO, M. (2007). Les facteurs psychosociaux à la base de la réussite universitaire et professionnelle. Aspects psychologiques et organisationnels. HDR en Psychologie. Université Lille 3 (Lille, France).

APARICIO, M. (2009a). Les facteurs psychosociaux et la réussite universitaire et professionnelle. ANRT (Lille, France).

\footnotetext{
${ }^{3}$ Même si dans le noyau qui nous occupe, le Monde du travail, les «compétences cognitives » n'émergent pas; il est important de souligner qu'il existe une différence entre les représentations partagées des français et des argentins par rapport à la valorisation des compétences pour agir actuellement : en France, globalement, sont priorisées les compétences sociocognitives ; en Argentine, plutôt, les compétences socio-affectives (typiquement valorisées dans les scenarios latinoaméricains).
} 
APARICIO, M. (2009b). Le devenir professionnel des étudiants qui sont engagés dans un doctorat en éducation/formation. Une approche comparative franco-argentine. Rapport. Prix de la Ville (Paris, France).

APARICIO, M. (2010). La evaluación de la Calidad del Sistema Universitario y de Empleo en su articulación ¿Hacia un paradigma sistémico transdisciplinario? Revista Iberoamericana de Educación, OEI (Madrid, España).

APARICIO, M. (2012). Trajectoires universitaires/professionnelles et identité. Formations et professionnalisations à l'épreuve de la complexité (195-229). In J. Clénet, Ph. Maubant et D. Poisson (Eds.). L'Harmattan (Paris, France).

APARICIO, M. (2015a). Towards a sui generis Systemic Theory: The Three-Dimensional Spiral of Sense. A Study in Argentina Applied to Identity and Professionalization (Article 1). Asian Academic Research Journal of Social Sciences \& Humanities, 2(8) : 246-282.

APARICIO, M. (2015b). The Theory of the Three-Dimensional Spiral of Sense: An Application with Special Reference to Identity and Professionalization in other Disciplinary Areas (Article 2). Asian Academic Research Journal of Social Sciences \& Humanities, 2(8) : 194-245.

APARICIO, M. \& CROS, F. (2015c). Trajectoires et identités. Un avenir incertain pour les docteurs? L'Harmattan (Paris, France).

Céreq. Nous avons consulté plusieurs ouvrages concernant la relation Formation-Emploi. En raison de l'espace, nous renvoyons au Rapport. Ville de Paris, 2009, op, cit.

DUBAR, C. (1991). La socialisation. Construction des identités sociales et professionnelles. Colin (Paris, France).

DUBAR, C. (1992). Formes identitaires et socialisation professionnelle. Revue Française de Sociologie, 33(4) : 505-529.

EDWARDS RISOPATRÓN, V. (1991). El concepto de calidad de la educación. UNESCO/OREALC (Santiago de Chile : Chile).

GUINGOUAIN, G. (1999). Psychologie sociale et évaluation. Dunod (Paris, France).

SANDER, B. (1990). Educación, administración y calidad de vida. Santillana (Buenos Aires, Argentine).

SILVA A-M. \& APARICIO M (Eds.), International Handbook about Professional Identities. Academic and Scientific Publishing (New York). 
